\title{
Convalescent plasma for pediatric patients with COVID-19 associated acute respiratory distress syndrome
}

\author{
Caroline Diorio $^{1}$, Elizabeth Anderson ${ }^{2}$, Kevin McNerney ${ }^{1}$, Eileen Goodwin ${ }^{2}$, Julie Chase ${ }^{1}$, \\ Marcus Bolton ${ }^{2}$, Claudia Arevalo ${ }^{2}$, Madison Weirick ${ }^{2}$, Sigrid Gouma ${ }^{2}$, Laura Vella ${ }^{1}$, Sarah \\ Henrickson $^{1}$, Kathleen Chiotos ${ }^{1}$, Julie Fitzgerald ${ }^{1}$, Todd Kilbaugh ${ }^{1}$, Audrey Odom John ${ }^{1}$, \\ Allison Blatz ${ }^{1}$, Michele Lambert ${ }^{1}$, Kathleen Sullivan ${ }^{1}$, Margaret Tartaglione ${ }^{1}$, Danielle \\ Zambrano $^{1}$, Meghan Martin ${ }^{1}$, Jessica Lee ${ }^{1}$, Pampee Young ${ }^{3}$, David Friedman ${ }^{1}$, Deborah \\ Sesok-Pizzini $^{1}$, Scott Hensley ${ }^{2}$, Edward Behrens ${ }^{1}$, Hamid Bassiri ${ }^{1}$, and David Teachey ${ }^{1}$ \\ ${ }^{1}$ The Children's Hospital of Philadelphia \\ ${ }^{2}$ University of Pennsylvania Perelman School of Medicine \\ ${ }^{3}$ American Red Cross
}

July 16, 2020

\begin{abstract}
There are no proven safe and effective therapies for children who develop life-threatening complications of SARS-CoV-2. Convalescent plasma $(\mathrm{CP})$ has demonstrated potential benefit in adults with SARS-CoV-2 but has theoretical risks. We report on the first use of CP in children with life-threatening COVID-19, providing data on four pediatric patients with acute respiratory distress syndrome. We measured donor antibody levels and recipient antibody response prior to and following CP infusion. Infusion of CP was not associated with antibody-dependent enhancement (ADE) and did not suppress endogenous antibody response. We found CP was safe and possibly efficacious. Randomized pediatric trials are needed.
\end{abstract}

\section{Introduction}

Early reports from the severe acute respiratory syndrome coronavirus 2 (SARS-CoV-2) global pandemic suggested that children were less severely affected by coronavirus disease (COVID-19) compared to older adults. ${ }^{1-3}$ Nevertheless, some children with COVID-19 are critically ill. ${ }^{4,5}$ Currently, no therapies have been proven effective in the treatment of COVID-19 in children.

Convalescent plasma (CP), derived from patients that have recovered from SARS-CoV-2 can be infused into currently ill patients. The proposed mechanism of action is via neutralizing antibodies binding to virus, rendering it inert. ${ }^{6}$ Specific to SARS-CoV-2, antibodies against the receptor binding domain (RBD) have been identified as surrogates for neutralization. ${ }^{7-11} \mathrm{CP}$ also contains antibodies against the immunogenic nucleocapsid $(\mathrm{N})$ protein, which are non-neutralizing and present in both actively infected and recovered patients. ${ }^{7}$ The protective function of $\mathrm{N}$ antibodies remains unclear. ${ }^{7,12}$ Antibodies against the full-length SARS-CoV-2 Spike (S) protein include both non-neutralizing antibodies and neutralizing antibodies. ${ }^{13}$

Initial series of $\mathrm{CP}$ in adults with COVID-19 demonstrated potential benefits without apparent side effects. ${ }^{14-16}$ In a recent clinical trial of adults with COVID-19, there was a non-statistically significant trend towards clinical improvement in CP-treated patients versus controls, without improvement in time to discharge or mortality. ${ }^{17}$ This trial was halted early due to poor accrual. ${ }^{17}$ To date, there have been no reports on the use of $\mathrm{CP}$ in children. 
$\mathrm{CP}$ infusion is associated with the side effects of any blood product: allergic reaction, transfusion-associated circulatory overload (TACO), and infection with blood borne pathogens. There is a theoretical risk that infusion of donor antibodies may impede the recipient's endogenous production of antibodies, and of antibodydependent enhancement (ADE), where antibodies developed during a previous infection cause a worsened clinical response with subsequent infection. ADE has been described in Dengue fever, and in pre-clinical models of other coronaviruses. ${ }^{18-20}$ ADE has not been reported in adult patients receiving CP for SARS$\mathrm{CoV} 2{ }^{6,17}$

We present the first report of pediatric patients receiving CP for life-threatening COVID-19 associated respiratory disease with correlative measurements of the associated pre- and post- transfusion antibody response.

\section{Methods}

We treated four critically ill children actively infected with SARS-CoV-2 with CP at the Children's Hospital of Philadelphia (CHOP) under emergency Investigational New Drug applications (eINDs) through the Food and Drug Administration (FDA). Patients were considered for CP if they met FDA guidance for life-threatening disease (Appendix S1). We prospectively screened and enrolled these patients into a larger SARS-CoV-2 biobanking protocol. Patients were enrolled on the biobank protocol if they had evidence of SARS-CoV-2 infection on reverse-transcriptase polymerase chain reaction (RT-PCR) testing from respiratory tract mucosa. This protocol was approved by the CHOP Institutional Review Board. Consent was obtained for both protocols from a legal authorized representative per the Declaration of Helsinki (written informed consent for CP; verbal consent for biobanking).

Clinical data were abstracted from patient charts to standardized forms by a research assistant using a REDCap database and verified by a physician. Blood samples were obtained from participants prior to $\mathrm{CP}$ infusion, following CP infusion and weekly thereafter until death or discharge. Remnant donor CP was retained.

SARS-CoV-2 specific antibodies were measured by ELISA as previously described. ${ }^{8}$ Antibodies in serially diluted plasma (starting at 1:50) were measured against RBD of the SARS-CoV-2 spike (S) protein, ${ }^{9}$ fulllength S protein, and the N protein (Sino Biological, Wayne, PA). Reciprocal plasma dilutions were calculated from an optical density (OD) threshold from a set concentration. Standard curves were generated by diluting an RBD-specific monoclonal antibody (CR3022) starting at $0.5 \mu \mathrm{g} / \mathrm{mL}$ (RBD and S ELISAs) or serially diluted pooled serum from actively SARS-CoV-2 infected adults (N ELISAs). See Appendix S2 for detailed methods.

\section{Results and Discussion}

Four patients, 14-18 years, were treated with CP during the study period. All patients were critically ill with COVID-19-associated acute respiratory distress syndrome (ARDS). All patients required intubation and ventilation; two required extra-corporeal membrane oxygenation (ECMO). None of the patients had Multisystem Inflammatory Syndrome in Children (MIS-C). All patients received other SARS-CoV-2 directed therapies in addition to CP. Graphical case summaries are presented in Figure 1. Further details are presented in Appendix S3. No patients experienced any treatment emergent adverse events (TEAE) related to CP infusion.

Longitudinal antibody titers are presented in Figure 2. Corresponding antibody titers for CP donors are displayed. At the time of CP infusion, patient CD4 had extremely high endogenous antibody titers (against SARS-CoV-2 RBD, S, and N). Antibody titers in this participant were sustained post-transfusion. This individual showed transient clinical improvement, decannulating from ECMO; however, died from cardiac causes by day 25 post-transfusion.

Patients CD15, CD17 and CD25 had low or undetectable RBD-specific antibody titers at the time of infusion. Serum antibodies against the SARS-CoV-2 RBD correlate with neutralizing activity, likely due to inhibition of the virus' ability to bind and enter target cells. ${ }^{8-10}$ Of note, the donor plasma infused into patients CD15 
and CD17 had relatively low RBD-specific antibody titer levels $(<1: 160)$. Both CP donors for CD15 and CD17 had substantial full-length IgG S titers $(>1: 1,000)$ and these may have conferred some neutralizing activity. It is unclear if $\mathrm{CP}$ with low RBD-specific and high full-length $\operatorname{IgG} \mathrm{S}$ antibody titers would provide benefit. CD15 and CD17 both remain in intensive care and did not have significant clinical improvement following CP infusion. The donor for patient CD25 had higher SARS-CoV-2 RBD antibody titers (>1:6,000) than the donor for any of the other patients, making it more likely that this donor plasma could be beneficial. CD25 had the shortest period of admission and has been discharged from the hospital after being critically ill and on ECMO.

Prior to transfusion, three of the four recipients (CD15, CD17, and CD25) had undetectable IgG RBD antibody levels $(<1: 50$ serum dilution). Upon transfusion all three participants eventually experienced a boost in IgG, IgM, and IgA antibodies against RBD that was sustained for 7-26 days post transfusion. In one participant (CD15), we detected antibodies against the full-length S protein before we detected antibodies against the RBD of the $\mathrm{S}$ protein. The boost in antibodies against $\mathrm{N}$ post-transfusion was similar to that of RBD and S-specific serum antibodies for patients CD15, CD17, and CD25. In all three patients, the serum levels of SARS-CoV-2-specific antibodies eventually surpassed the amount transfused, indicating that these patients mounted a post-transfusion de novo response and that $\mathrm{CP}$ did not eliminate the endogenous serological response.

In summary, CP was safely administered to four pediatric patients with severe COVID-19. There were no CP-related TEAE. The theoretical risks of ADE or elimination of endogenous antibody response did not occur. CP may be of greatest benefit for patients who are early in their illness and have not yet generated endogenous antibodies, and when the infused plasma has a high antibody titer. The patient who received the $\mathrm{CP}$ with the highest antibody titer had the best clinical response. The small sample size of our study precludes definitive conclusions about efficacy, however, the excellent clinical response in this patient is encouraging. The other patients had significant comorbidities that may have contributed to their clinical status. It is difficult to determine if CD15 and CD17 benefited, as it is possible without CP they could have continued to worsen. Future research should include randomized controlled trials to definitively examine the efficacy of convalescent plasma in children.

\section{Authorship contributions}

CD and EMA equally contributed to this work and are senior first authors. EMB, DTT, and HB equally contributed to this work and are co-senior authors. CD, EA, SEH, LV, KOM, JC, SH, EB, HB, and DTT designed the study, analyzed data, and wrote the manuscript; KC, ML, AMB, JCF, TJK, AROJ, KES, DF, DASP, MRT, DZ, MM, JHL, PY contributed to study design, analyzed data, and helped write the manuscript. EMA, MEW, SG, EC, MJB, CPA performed experiments, provided data, and edited manuscript. CD, JC, and KOM enrolled subjects. PY, DSP and DF also provided specimens. All authors contributed intellectually and reviewed and revised the manuscript.

\section{Acknowledgements and Financial Support}

Financial support for this project was provided by CHOP Frontiers Program Immune Dysregulation Team (DTT, EB, HB), National Institute of Allergy and Infectious Diseases (NIAID) (US): R01AI121250 (EMB), R01AI103280 (AOJ), R01AI123433 (AOJ), R21AI144472 (AOJ), K08 AI136660 (LVV), and K08AI135091(SH), Agency for Healthcare Research and Quality (AHRQ) 5K12HS026393 (KC), National Cancer Institute (NCI) (US): R01CA193776 (DTT), X01HD100702-01(DTT), 5UG1CA233249) (DTT), and R01A1123538 (DTT), NIH Training in Virology T32-AI-007324 (EMA), Leukemia and Lymphoma Society (DTT), Cookies for Kids Cancer (DTT), Alex's Lemonade Stand Foundation for Childhood Cancer (DTT), Children's Oncology Group (DTT), Stand UP 2 Cancer (DTT), Team Connor (HB), and Kate Amato Foundations (HB). Burroughs Wellcome Fund CAMS (SH) and PATH (AOJ), Clinical Immunology Society (SH), and American Academy of Allergy, Asthma, and Immunology (SH). Serological studies were supported by institutional funds from the University of Pennsylvania. CD was supported by the Institute for Translational Medicine and Therapeutics of the Perelman School of Medicine at the University of Pennsylvania. We 
gratefully acknowledge the gift of plasmids of the receptor binding domain (RBD) of the SARS-CoV-2 spike (S) protein from Florian Krammer, Mt. Sinai and the gift of the RBD-specific monoclonal antibody CR3022 from Ian Wilson, Scripps Research Institute. We gratefully acknowledge the providers who cared for these patients, and the generosity of the patients and their families.

\section{Conflicts of Interest}

No author has a relevant conflict of interest. DTT serves on advisory boards for Janssen, Amgen, La Roche, Sobi, and Humanigen. HB has stock ownership in CSL Behring. SH serves on the advisory board for Horizon Pharma. AOJ serves on the advisory board of Pluton Biosciences. MPL is an advisory board member for Octapharma, Novartis, Aregenix, and Shionogi, a consultant for Amgen, Novartis, Shionogi, Dova, Bayer, and has received research funding from Sysmex, Novartis, and Astra Zeneca. KES received personal fees from Elsevier and Immune Deficiency Foundation. SEH has received consultancy fee from Sanofi Pasteur, Lumen, Novavax, and Merck for work unrelated to this report.

\section{References}

1. Dong Y, Mo X, Hu Y, et al. Epidemiological characteristics of 2143 pediatric patients with 2019 coronavirus disease in China. Pediatrics . 2020:e20200702.

2. Liu W, Zhang Q, Chen J, et al. Detection of Covid-19 in children in early January 2020 in Wuhan, China. N Engl J Med . 2020;382(14):1370-1.

3. Huang C, Wang Y, Li X, et al. Clinical features of patients infected with 2019 novel coronavirus in Wuhan, China. Lancet . 2020;395(10223):497-506.

4. Zachariah P, Johnson CL, Halabi KC, et al. Epidemiology, Clinical Features, and Disease Severity in Patients With Coronavirus Disease 2019 (COVID-19) in a Children's Hospital in New York City, New York. JAMA Pediatr . 2020:e202430.

5. Chao JY, Derespina KR, Herold BC, et al. Clinical Characteristics and Outcomes of Hospitalized and Critically Ill Children and Adolescents with Coronavirus Disease 2019 (COVID-19) at a Tertiary Care Medical Center in New York City. J Pediatr . 2020; doi: https://doi.org/10.1016/ j.jpeds.2020.05.006.

6. Bloch EM, Shoham S, Casadevall A, et al. Deployment of convalescent plasma for the prevention and treatment of COVID-19. J Clin Invest . 2020; 2757-2765.

7. Hachim A, Kavian N, Cohen CA, et al. Beyond the Spike: identification of viral targets of the antibody response to SARS-CoV-2 in COVID-19 patients. medRxiv . 2020:2020.2004.2030.20085670.

8. Flannery D, Gouma S, Dhudasia M, et al. SARS-CoV-2 Seroprevalence Among Parturient Women. https://www.researchsquare.com/article/rs-27402/v1; 2020.

9. Amanat F, Stadlbauer D, Strohmeier S, et al. A serological assay to detect SARS-CoV-2 seroconversion in humans. Nat Med . 2020;1-4.

10. Okba NM, Müller MA, Li W, et al. Severe Acute Respiratory Syndrome Coronavirus 2-Specific Antibody Responses in Coronavirus Disease 2019 Patients. Emerg Infect Dis . 2020;26(7).

11. Premkumar L, Segovia-Chumbez B, Jadi R, et al. The receptor binding domain of the viral spike protein is an immunodominant and highly specific target of antibodies in SARS-CoV-2 patients. Sci Immunol . $2020 ; 5(48)$.

12. Leung DT, Tam FC, Ma CH, et al. Antibody response of patients with severe acute respiratory syndrome (SARS) targets the viral nucleocapsid. J Infect Dis . 2004;190(2):379-386.

13. Lv H, Wu NC, Tsang OT, et al. Cross-reactive Antibody Response between SARS-CoV-2 and SARS-CoV Infections. Cell Rep . 2020;31(9):107725. 
14. Shen C, Wang Z, Zhao F, et al. Treatment of 5 Critically Ill Patients With COVID-19 With Convalescent Plasma. JAMA . 2020; 323(16): 1582-1589.

15. Zhang B, Liu S, Tan T, et al. Treatment With Convalescent Plasma for Critically Ill Patients With SARS-CoV-2 Infection. Chest . 2020. https://doi.org/10.1016/j.chest.2020.03.039

16. Duan K, Liu B, Li C, et al. Effectiveness of convalescent plasma therapy in severe COVID-19 patients. Proc Natl Acad Sci U S A . 2020; 117(17): 9490-9496

17. Li L, Zhang W, Hu Y, et al. Effect of Convalescent Plasma Therapy on Time to Clinical Improvement in Patients With Severe and Life-threatening COVID-19: A Randomized Clinical Trial. JAMA . 2020; doi:10.1001/jama.2020.10044

18. Yip MS, Leung NHL, Cheung CY, et al. Antibody-dependent infection of human macrophages by severe acute respiratory syndrome coronavirus. Virol $J$. 2014;11(1):82.

19. Wang S-F, Tseng S-P, Yen C-H, et al. Antibody-dependent SARS coronavirus infection is mediated by antibodies against spike proteins. Biochem Biophys Res Comun . 2014;451(2):208-214.

20. Agrawal AS, Tao X, Algaissi A, et al. Immunization with inactivated Middle East Respiratory Syndrome coronavirus vaccine leads to lung immunopathology on challenge with live virus. Hum Vacc Immunother . 2016;12(9):2351-2356.

\section{Figures and Figure Legends}

Figure 1. Graphical representation of clinical course of four patients included in the case series. Timeline includes up to day 40 of illness, or until death or discharge, whatever occurred first. PCR - polymerase chain reaction; HCQ - hydroxychloroquine; ECMO - extracorporeal membrane oxygenation.

Figure 2. Longitudinal serum SARS-CoV-2 antibody titers in pediatric patients receiving convalescent plasma transfusion therapy. Antibody titers expressed as reciprocal serum dilution against SARS-CoV-2 antigens in four pediatric patients (CD4, CD15, CD17 and CD25) and corresponding convalescent plasma donors (CP; open symbols) before and after plasma transfusion. Antibody titers against (a-d) the SARS-CoV-2 receptor binding domain of the spike (S) protein, (e-h) the full-length SARS-CoV-2 S protein (FL), and (i-l) the nucleoprotein of SARS-CoV-2 (N). Dashed lines denote the lower limit of detection (LLOD) of the assay (1:50 serum dilution)

Figure 1. Graphical representation of clinical course of four patients included in the case series. Timeline includes up to day 40 of illness, or until death or discharge, whatever occurred first. PCR - polymerase chain reaction; HCQ - hydroxychloroquine; ECMO - extracorporeal membrane oxygenation.

\section{Hosted file}

image1.emf available at https://authorea.com/users/343383/articles/470053-convalescentplasma-for-pediatric-patients-with-covid-19-associated-acute-respiratory-distresssyndrome

Figure 2. Longitudinal serum SARS-CoV-2 antibody titers in pediatric patients receiving convalescent plasma transfusion therapy.Antibody titers expressed as reciprocal serum dilution against SARS-CoV-2 antigens in four pediatric patients (CD4, CD15, CD17, and CD25) and corresponding convalescent plasma donors (CP; open symbols) before and after plasma transfusion. Antibody titers against (a-d) the SARS-CoV-2 receptor binding domain of the spike protein (RBP), (e-h) the full-length SARS-CoV-2 S protein $(\mathrm{S})$, and (i-l) the nucleocapsid protein of SARS-CoV-2 $(\mathrm{N})$. Dashed lines denote the lower limit of detection (LLOD) of the assay (1:50 serum dilution)

\section{Hosted file}

image9.emf available at https://authorea.com/users/343383/articles/470053-convalescentplasma-for-pediatric-patients-with-covid-19-associated-acute-respiratory-distress- 
syndrome 